\section{Bone turnover markers in patients with type 1 Gaucher disease}

\author{
Gaetano Giuffrida, Maria Rocca Cingari, \\ Nunziatina Parrinello, \\ Alessandra Romano, Anna Triolo, \\ Magda Franceschino, \\ Francesco Di Raimondo
}

Department of Clinical and Molecular

Biomedicine, Division of Hematology,

University of Catania, Ospedale

Ferrarotto, Catania, Italy

\section{Abstract}

Bone complications occur frequently in Gaucher disease (GD) and reduce the quality of life of these patients. Skeletal involvement is an important indication for treatment to ameliorate symptoms and reduce the risk of irreversible and debilitating disease. Bone biomarkers have been used to assess disease status and the response to therapy in a number of bone disorders. Here, we examine the literature for evidence of abnormalities in bone turnover markers in patients with type $1 \mathrm{GD}$ to assess whether they might be useful for the assessment of bone involvement in GD. We have found that bone biomarkers in GD show highly variable results which do not currently support their routine use for clinical assessment of bone status, as an indication for therapy initiation, or for monitoring the response to therapy. A greater understanding of bone markers and their relation to the bone manifestations of GD is required.

\section{Introduction}

Gaucher Disease (GD) is a rare autosomal recessive hereditary disorder of glycosphingolipid metabolism, characterized by the accumulation of glucosylceramide in cells of the reticulo-endothelial system, due to deficient activity of the lysosomal enzyme, beta-glucosidase (glucocerebrosidase, EC 3.2.1.45). There are various manifestations of GD but the disease is generally described by three main phenotypes: type 1 GD (GD1), which can manifest at any age and is distinguished by a lack of primary neurological manifestations, and type 2 (acute) and type 3 (chronic) neuronopathic phenotypes which manifest in early life, involve the central nervous system, and result in early death. usually in infancy (type 2) and childhood or early adulthood (type 3 ). ${ }^{1}$

Type $1 \mathrm{GD}$ is, by far, the most common form of GD. The age of onset, rate of disease progression, severity of symptoms, and the organs affected can vary widely. Some patients with GD1 may remain asymptomatic although the majority experience symptoms that commonly include fatigue, splenomegaly, anemia, thrombocytopenia, hepatomegaly, and skeletal disease. The skeletal manifestations of GD, which may lead to significant pain, morbidity, disability and a progressive reduction in quality of life,$^{2}$ include abnormal bone remodeling, osteopenia, osteoporosis, lytic lesions, avascular necrosis, pathological fractures, osteonecrosis of humeral or femoral heads, and vertebral collapse. ${ }^{3}$ Osteopenia is a common and often early manifestation of Gaucher-related bone disease which may lead to reduced bone mineral density (BMD), reduced peak bone mass, fragility fracture and poor bone healing. ${ }^{4}$

Treatment for the systemic manifestations of GD has been available for over 20 years in the form of enzyme replacement therapy, firstly as alglucerase (Ceredase; Genzyme Corporation, Cambridge, MA, USA), and subsequently by the recombinant form imiglucerase (Cerezyme, Genzyme Corporation, Cambridge, MA, USA). ${ }^{5}$ In more recent years, an alternative treatment, substrate reduction therapy, has also become available for adult patients who are unwilling or unable to be treated with enzyme therapy. ${ }^{6}$ Another enzyme replacement therapy has also become available: velaglucerase-alfa (Shire Human Genetic Therapies). ${ }^{7}$ Before diseasespecific treatment became available for Gaucher disease, splenectomy was frequently carried out to control bleeding risk and the mechanical effects of an enlarged spleen in severely affected patients. It is now known that splenectomy may be associated with an exacerbation of GD-related bone disease..$^{8,9}$

Skeletal involvement is an important indication for treatment to ameliorate symptoms, prevent osteoporosis and reduce risk of pathological fractures. ${ }^{4,10}$ While some skeletal symptoms of GD, such as bone pain and bone crises, may be ameliorated within 1-2 years by treatment with imiglucerase enzyme replacement therapy, it is recognized that the response of some bone manifestations, such as BMD, to treatment can be slower, ${ }^{10,11}$ and some bone complications, such as avascular necrosis, are irreversible despite optimal treatment. ${ }^{12}$ This underlines the need for early initiation of therapy if the risk of irreversible complications is to be reduced. It is known that BMD is accrued mostly in the first 20 years of life, peaks in the third decade, and then declines, suggesting that there is a limited therapeutic window in which to gain maximal benefit from treatment. ${ }^{13}$ Similarly, it has been shown that the risk of avascular necrosis (AVN) appears to be reduced in patients who initiate enzyme replacement therapy within two years of diag-
Correspondence: Gaetano Giuffrida, Department of Clinical and Molecular Biomedicine, Division of Hematology, University of Catania, Ospedale Ferrarotto, Catania, Italy.

Tel. +39.095.7435923 - Fax: +39.095.365174.

E-mail: gagimapi@infinito.it

Key words: Gaucher disease, bone, biomarkers, osteopenia.

Conflict of interests: the preparation of this manuscript was supported by an unrestricted educational grant from Genzyme Corporation, which included funding for English language medical writing support from P. Pickering, Conscience Creative LLP, UK. The opinions of the authors are their own and do not necessarily reflect those of Genzyme Corporation. The authors declare that they have no further conflict of interests.

Received for publication: 13 December 2011. Revision received: 3 July 2012.

Accepted for publication: 10 September 2012.

This work is licensed under a Creative Commons Attribution NonCommercial 3.0 License (CC BYNC 3.0).

(C) Copyright G. Giuffrida et al., 2012

Licensee PAGEPress, Italy

Hematology Reports 2012; 4:e21

doi:10.4081/hr.2012.e21

nosis compared with those initiating therapy after two years, ${ }^{14}$ although the risk of AVN may not be eliminated in patients receiving enzyme therapy. ${ }^{12}$

One of the challenges of monitoring bone involvement and treating bone manifestations is that the pathophysiology of bone disease is not completely understood. Although the infiltration of Gaucher cells into the bone marrow and bone is thought to be a central event in the pathophysiological process, ${ }^{15}$ it is unclear whether, and to what extent, complications are due to mechanical effects, such as compression, and/or the indirect effects of Gaucher cells on bone (through the activation of inflammatory mediators), or whether GD alters bone homeostasis independently of Gaucher cells. ${ }^{16}$

The Erlenmeyer flask deformity has been shown to be present in up to $59 \%$ of GD patients, ${ }^{12}$ suggesting that bone remodeling disorders are common in this disease and may have relevance to skeletal pathologies. Bone turnover or remodeling in healthy individuals is a lifelong process by which mature bone is resorbed (mediated by osteoclasts) and new bone tissue is formed (mediated by osteoblasts followed by calcification). 0steoclasts are recruited from bone marrow precursors which attach to the surface of bone and break down 
the bone matrix through the release of proteolytic enzymes to leave surface pits. Osteoblasts differentiate from connective tissue precursors and fill the bone pits with a protein matrix which subsequently becomes fully mineralized bone. ${ }^{17}$ The biochemical processes involved in bone turnover result in measurable changes in enzyme activities, the release of various bone components, and the expression of proteins involved in the mineralization of the bone matrix. These biomarkers of bone turnover are outlined in Table 1.

The activity of osteoclasts and osteoblasts is normally tightly coupled, but in several pathological states and with increasing age the turnover process may be disrupted. ${ }^{17}$ Signaling networks between osteoclasts and osteoblasts are central to the regulation of bone turnover. ${ }^{18}$ Osteoclasts, for example, have a role in regulating the activity of other cells, including osteoblast precursors, by participating in immune responses and by secreting cytokines that may affect their own function and those of other cells. ${ }^{19}$ Osteoclast formation, in turn, is dependent on the cytokine RANKL through binding to its cognate receptor RANK on the surface of osteoclast precursors.
Osteoprotegerin (OPG), a decoy receptor for RANKL, inhibits osteoclast differentiation and osteoclast activity. ${ }^{20}$ The interaction between OPG and RANKL, therefore, has a role in regulating osteoclast formation and provides an additional means of estimating bone turnover. ${ }^{21,22}$ The OPG/RANKL system is of particular interest because it has been exploited as a therapeutic target in some bone diseases. ${ }^{23,24}$

Bone biomarkers have been used to assess disease status and the response to therapy in a number of bone disorders, ${ }^{25}$ including postmenopausal osteoporosis, ${ }^{26}$ Paget's disease, ${ }^{27}$ rheumatoid arthritis, ${ }^{28,29}$ and cancer. ${ }^{30}$ Here, we examine the literature for evidence of abnormalities in markers of bone turnover in patients with type $1 \mathrm{GD}$. While biomarkers of activated macrophages, such as chitotriosidase, are often used to assess the whole body burden of Gaucher cells and the whole body response to therapy, there is currently no recommended biomarker that is specific to bone. ${ }^{8}$ Skeletal disease in GD may develop silently, may not correspond with systemic disease in terms of onset, severity or rate of progression, and may not always be detectable by the com- monly used imaging methods. ${ }^{3,12}$ Some bone imaging methods, especially the more sensitive techniques such as quantitative chemical shift imaging (QCSI), are expensive, complex and not widely available for routine monitoring. ${ }^{3}$ The identification of a sensitive biomarker specific for bone disease in GD would, therefore, be of significant benefit for detecting and monitoring bone involvement, and would help clinicians determine optimal timing of treatment for the prevention of bone complications.

The available English language literature on bone markers in GD was reviewed using online search methods such as PubMed. Literature was not restricted by date of publication.

Thirteen studies involving the measurement of bone turnover markers in GD1 patients were identified. One of these studies also included data from patients with type 3 disease. ${ }^{31}$ A summary of results from all studies is presented in Table 2.31-45 An overview of biomarkers in GD patients who were untreated, treated with enzyme therapy, and treated with bisphosphonates is to be found in Table $3 .^{34-44}$

Table 1. Bone biomarkers used to assess bone turnover.

\begin{tabular}{|c|c|c|c|}
\hline \multicolumn{4}{|c|}{$\begin{array}{lc} & \text { Markers of bone formation } \\
\text { Marker } & \text { Source }\end{array}$} \\
\hline Carboxyterminal propeptide of type 1 procollagen (PICP) & Pro-collagen & Weak (also in soft tissue) & Serum \\
\hline Amino (N-)terminal propeptide of type 1 procollagen (PIN & Pro-collagen & Weak (also in soft tissue) & Serum \\
\hline Osteocalcin $(\mathrm{OC})$ & $\begin{array}{l}\text { Protein produced by mature } \\
\text { osteoblasts in bone matrix }\end{array}$ & $\begin{array}{l}\text { High. The major non collagen protein } \\
\text { of bone secreted by osteoblasts. } \\
\text { Osteocalcin fragments also released } \\
\text { ring resorption. Reflects both bone formation } \\
\text { and bone resorption. }\end{array}$ & Serum \\
\hline Total alkaline phosphatase (AP) & Osteoblast enzyme & $\begin{array}{l}\text { Low: Present in plasma membrane } \\
\text { of osteoblasts but also liver, kidney, } \\
\text { intestinal, and spleen cells }\end{array}$ & Serum \\
\hline Bone specific alkaline phosphatase (BSAP) & Osteoblast enzyme & $\begin{array}{l}\text { Highly specific for bone - but up } \\
\text { to } 20 \% \text { cross reactivity between bone } \\
\text { and liver enzymes }\end{array}$ & Serum \\
\hline \multicolumn{4}{|c|}{ Markers of bone resorption } \\
\hline Pyridinolines (free and total pyr) & Collagen breakdown & Specific (primarily bone and cartilage) & Urine \\
\hline $\begin{array}{l}\text { Deoxy-pyridinolines (D-pyr) (also known as D-Pyrilinks, } \\
\text { Pyrilinks-D, or deoxyPYD) }\end{array}$ & Collagen breakdown & Highly specific (primarily bone and cartilage) & Urine \\
\hline Hydroxyproline (HP) & Collagen breakdown & Weak (found in bone, soft tissue, diet) & Urine \\
\hline Carboxyterminal telopeptide of type 1 collagen (ICTP) & Collagen breakdown & Highly specific (primarily bone) & Serum \\
\hline $\begin{array}{l}\text { Cross-linked type } 1 \text { collagen } \\
\text { C-terminal telopeptide (CTX) } \\
\text { Amino (N-)terminal telopeptide (NTX) }\end{array}$ & Collagen breakdown & Highly specific (primarily from bone) & $\begin{array}{l}\text { Serum and } \\
\text { urine }\end{array}$ \\
\hline $\begin{array}{l}\text { Cross-linked type } 1 \text { collagen } \\
\text { C-terminal telopeptide (CTX) } \\
\text { N-terminal telopeptide (NTX) }\end{array}$ & Collagen breakdown & Highly specific (primarily from bone) & $\begin{array}{l}\text { Serum and } \\
\text { urine }\end{array}$ \\
\hline $\begin{array}{l}\text { Tartrate-resistant acid phosphatase (TRAP) } \\
\text { (TRAP5b) }\end{array}$ & Osteoclast enzyme & $\begin{array}{l}\text { TRAP also found in other tissues } \\
\text { Specific for osteoclasts }\end{array}$ & Serum \\
\hline Cathepsin K & Proteolytic enzyme & Specific for bone osteoclasts & Serum \\
\hline $\mathrm{NF}_{-\mathrm{K}} \mathrm{B}$ ligand (RANKL) and osteorotegerin (OPG) & Osteoclast recruitment and activity & Osteoclasts & Serum \\
\hline
\end{tabular}




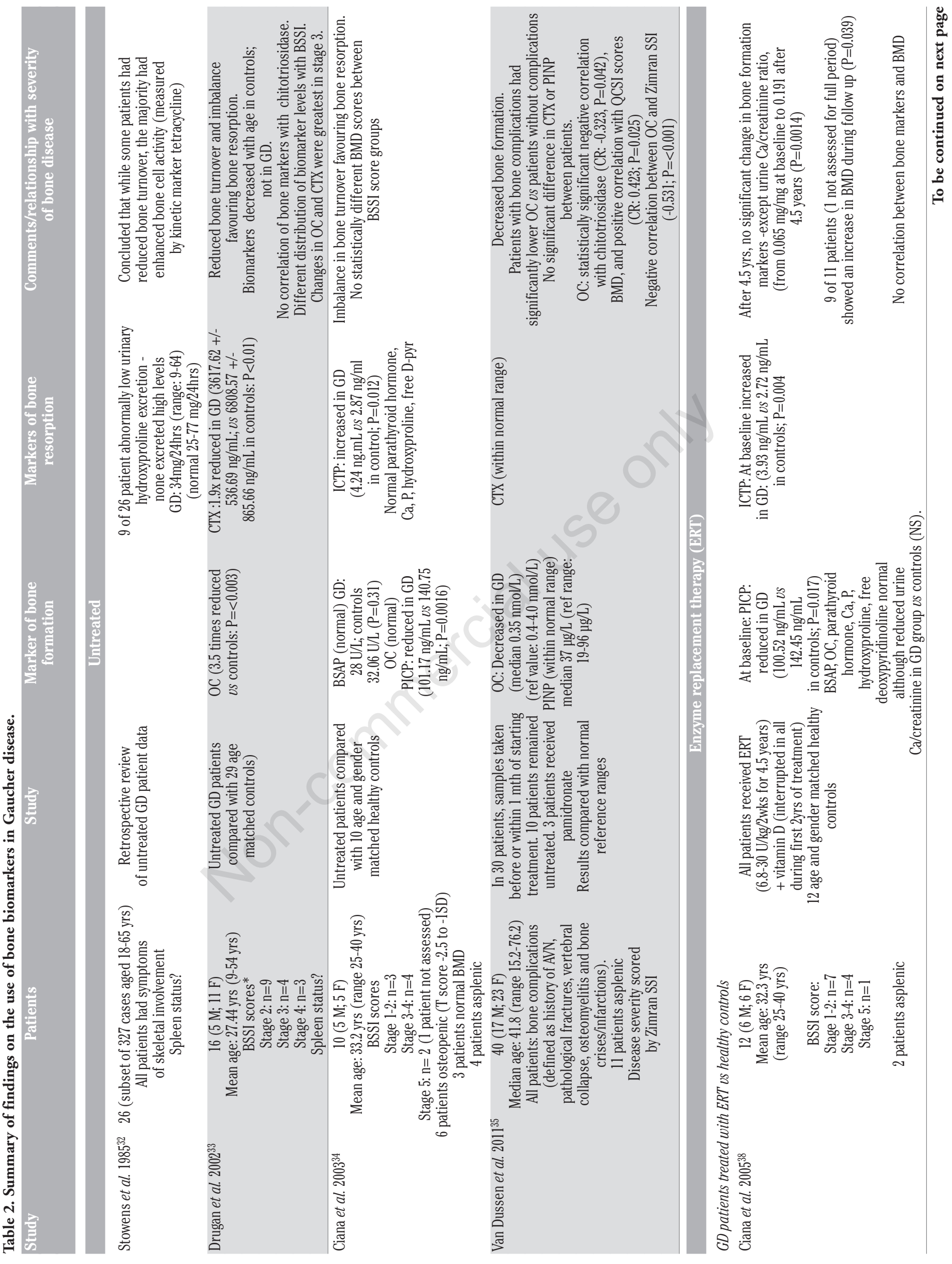




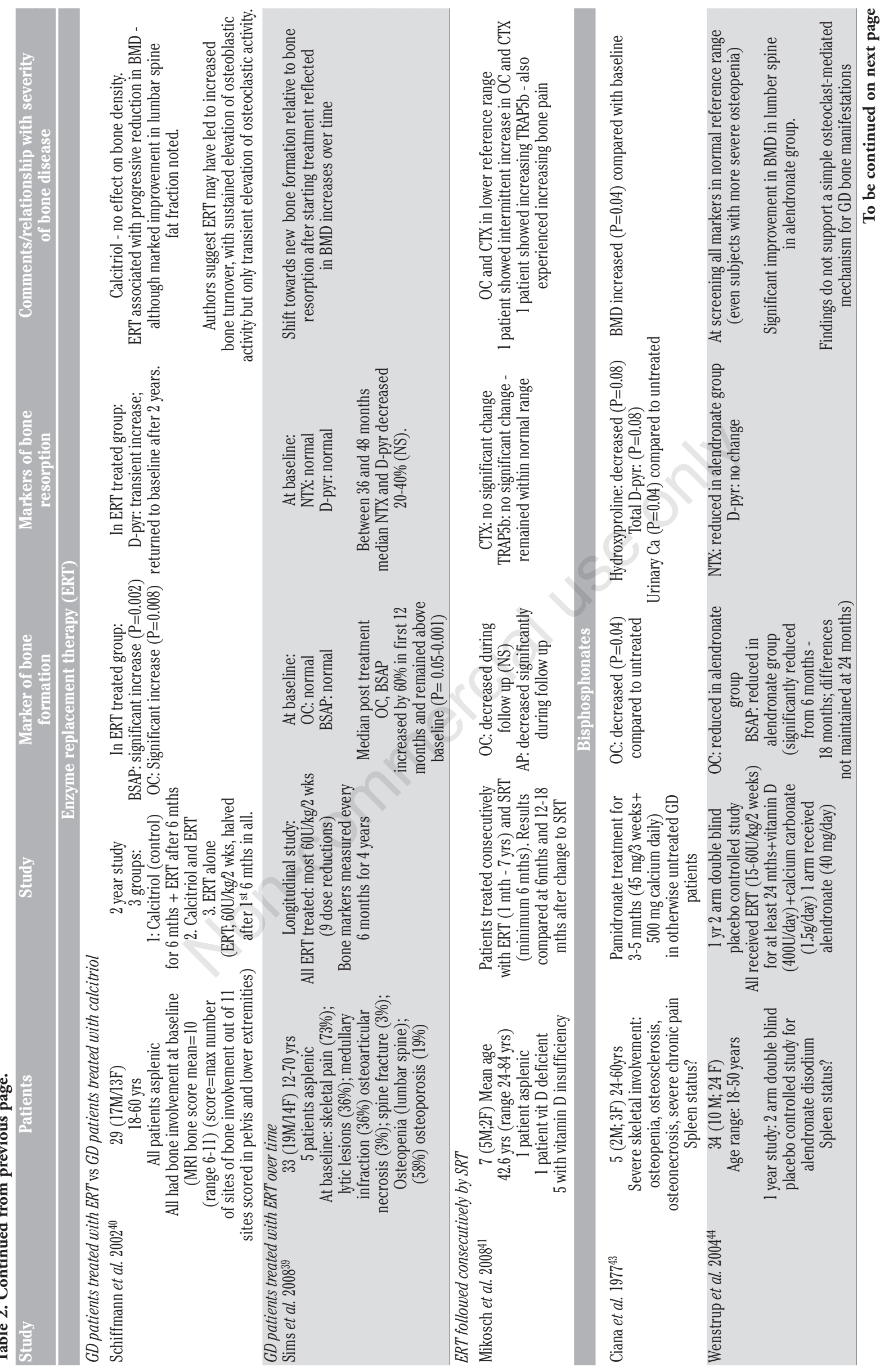




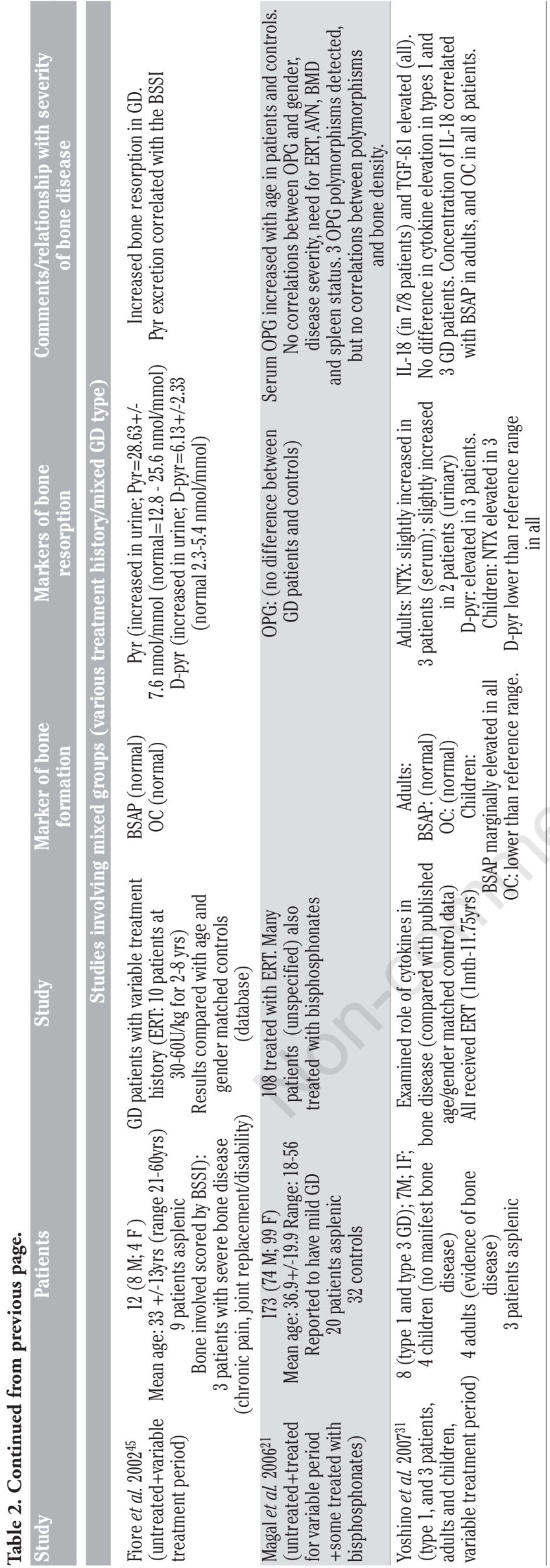

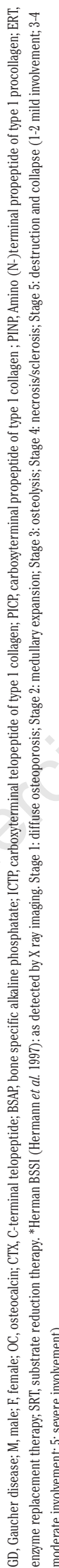

\section{Bone biomarkers in untreated Gaucher patients}

Three studies were carried out in untreated Gaucher patients in which results were compared with healthy control data. ${ }^{32-34}$ One study examined bone biomarkers in patients from whom samples were taken before or within one month of starting enzyme therapy, ${ }^{35}$ considered here as a study of untreated patients.

Of the studies examining bone markers in treatment-naïve patients, Stowens et al. ${ }^{32}$ found decreased hydroxyproline excretion in 9 of 26 patients (35\%) compared with controls, suggesting reduced bone turnover (resorption) in these patients. Reduced bone turnover was also found in a study involving 16 untreated Gaucher patients, ${ }^{33}$ in whom there was a 3.5 times decrease in osteocalcin levels and a 1.9 times decrease in type 1 collagen $\mathrm{C}$ terminal telopeptide (CTX), suggesting reduced osteoblastic and osteoclastic activity but with a net imbalance in bone turnover favoring bone resorption. No correlation was found between bone markers and genotype, chitotriosidase activity, or the Zimran Severity Score Index (SSI) for GD. ${ }^{36}$ A significant difference was, however, noted in serum osteocalcin levels and CTX according to the stage of skeletal involvement, as assessed by the Hermann Bone Severity Score Index, ${ }^{37}$ with both markers exhibiting the highest levels in stage 3 disease. A reduction in bone biomarker levels in the more advanced stage 4 disease was suggested to be related to an extended loss of functional bone tissue due to osteonecrosis, with a marked decline in both osteoblast and osteoclast activity.

A further study involving $10 \mathrm{GD}$ patients also concluded that there was increased bone resorption in GD compared with healthy controls based on a decrease in carboxyterminal propeptide of type I procollagen (PICP) and an increase in carboxyterminal telopeptide of type 1 collagen (ICTP) while osteocalcin was unchanged. ${ }^{38}$ No differences were found in hydroxyproline or free deoxypyridinoline (Dpyr; a more sensitive marker unaffected by collagen synthesis or dietary collagen) or the urinary calcium/creatinine ratio between Gaucher patients and controls. There was no correlation between bone marker levels and patient demographics, splenectomy status, spleen and liver volume, or bone mineral density.

Van Dussen et al. ${ }^{35}$ found that osteocalcin was decreased in $50 \%$ of GD1 patients $(n=40)$ with no significant change in type 1 collagen CTX, a marker of bone resorption, and concluded that imbalances in bone turnover result primarily from a decrease in bone formation. Osteocalcin levels showed a statistically significant negative correlation with chitotriosidase 
activity, a positive correlation with QCSI scores $(-0.323, \mathrm{P}=0.042$; and $0.423, \mathrm{P}=0.025$, respectively) and other bone turnover markers PINP and CTX (0.483, $\mathrm{P}=0.002$; and $0.466, \mathrm{P}=0.002$, respectively). Osteocalcin also showed a negative correlation with the Zimran SSI score ($0.531, \mathrm{P}<0.001)$. The correlations between SSI values and QCSI scores remained significant when the possible confounding contribution of patients receiving bisphosphonates (3 of 40 patients, 7.5\%) or who had a recent bone crisis (4 of 40 patients, 10\%) were excluded. There was also a negative correlation between osteocalcin and BMD in a subset of patients relatively mildly affected ( $\mathrm{n}=12 ; \mathrm{r}=-0.639$; $\mathrm{P}=0.885 ; \mathrm{P}<0.001$ ) but no statistically significant correlation between osteocalcin and the bone marrow burden score $(-0.382 ; \mathrm{P}=0.08)$.

In an analysis of pre-treatment data cited by studies investigating bone marker levels during treatment, Sims et al. ${ }^{39}$ found normal biomarker levels [osteocalcin, bone specific alkaline phosphatase (BSAP), type 1 collagen $\mathrm{N}$ terminal telopeptide (NTX) and D-pyr] at baseline while Ciana et al. found significantly reduced PICP and significantly increased ICTP compared with controls. ${ }^{38}$

\section{Bone biomarkers in response to enzyme replacement therapy}

Studies involving enzyme replacement therapy differed in purpose and design. One study examined bone biomarkers over time in enzyme-treated patients in whom bone markers were measured every six months for four years. ${ }^{39}$ Another looked at markers in enzymetreated patients (who also received interrupted vitamin D supplementation) treated for 4.5 years compared with healthy controls. ${ }^{38}$ Schiffmann et al. ${ }^{40}$ compared bone markers in 29 splenectomized patients treated with either enzyme therapy alone or enzyme therapy and calcitriol (1,25-dihydroxyvitamin $\left.\mathrm{D}_{3}\right)$, and Mikosch et al. ${ }^{41}$ examined biomarkers in patients treated with enzyme therapy (one month to seven years) followed consecutively by substrate reduction therapy with follow up for up to 18 months.

Ciana et al. ${ }^{38}$ found no significant change in bone markers over 4.5 years after the initiation of enzyme replacement therapy except for a statistically significant increase in the calcium/creatinine urinary ratio. Patients showed a gradual improvement in BMD over the 4.5 years which was not reflected in bone biomarker levels.

Sims et al. ${ }^{39}$ found that the markers of bone formation (osteocalcin and BSAP) increased by approximately $60 \%$ in the first 12 months after the initiation of enzyme replacement therapy (without any other medications) and
Table 3. Overview of biomarker activity in untreated Gaucher disease (GD) patients, GD patients treated with enzyme replacement therapy, and GD patients treated with bisphosphonates (excluding studies involving both untreated and treated patients).

\begin{tabular}{|c|c|c|c|}
\hline Marker & Normal & $\begin{array}{l}\text { Untreated } \\
\text { Increased }\end{array}$ & Decreased \\
\hline
\end{tabular}

\section{Formation}

BSAP

\begin{tabular}{lccc} 
OC & {$[34]$} & {$[33]$} & {$[35]$} \\
& {$[39]^{*}$} & {$[34]$} \\
& {$[38]^{*}$} & {$[38]^{*}$} \\
\hline PICP & & \\
PINP & {$[35]$} & & \\
\hline Resorption & {$[34]$} & {$[33]$} \\
\hline Calcium/creatinine & {$[35]$} & {$[32]$} \\
CTX & {$[34]$} & {$[34]^{*}$} \\
\hline Hydroxyproline & {$[34]$} & {$[39]$} & \\
D-pyr & & \\
\hline ICTP & {$[39]$} & \\
NTX & & \\
\hline
\end{tabular}

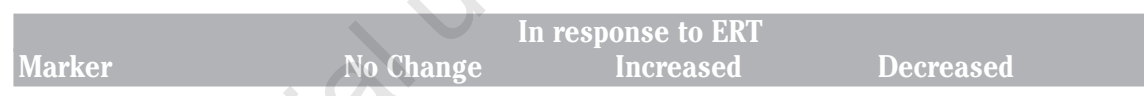

$\begin{array}{ll}\text { Formation } & \\ \text { BSAP } & {[40]}\end{array}$

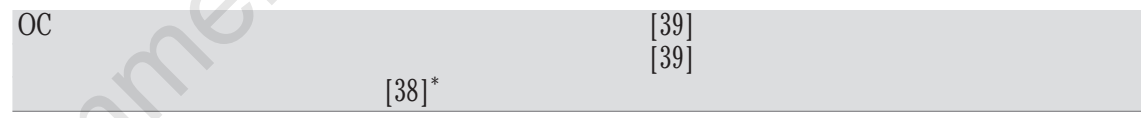
PICP
$[38]^{*}$

Resorption

Calcium/creatine

$[38]^{*}$

Hydroxyproline

ICTP

[38]

$[38]^{*}$

In response to bisphosphonates
No change $\quad$ Increased

\section{Formation}

BSAP

[44]

OC

[43]

Resorption

D-pyr

$[44]$

\begin{tabular}{ll} 
Hydroxyproline & [43] \\
\hline NTX & {$[44]$}
\end{tabular}

*7 of 12 patients included in the study by Ciana et al 200538 were also included in the study by Ciana et al. 2003 ${ }^{34}$ ERT, enzyme replacement therapy; BSAP, Bone specific alkaline phosphatase; CTX, Type 1 collagen C-terminal telopeptide; D-pyr, Deoxy-pyridinolines; ICTP, Carboxyterminal telopeptide of type 1 collagen; NTX, Amino (N-)terminal telopeptide of type 1 collagen; PICP, Carboxyterminal propeptide of type 1 procollagen; PINP, Amino (N-)terminal propeptide of type 1 procollagen. 
remained above baseline for the remainder of the study (four years). At least $74 \%$ of patients showed increases in these markers over baseline. There was no significant change in markers of bone resorption. Statistically significant improvements in BMD occurred over the 4 -year study period. Patients reported a reduction in bone pain and bone crises. At baseline, 13 of 33 patients had a history of bone crises; 2 patients reported bone crises in the first year, one in the second year, and none thereafter.

In a study involving splenectomized patients, BSAP and osteocalcin increased significantly after the initiation of enzyme therapy while there was a transient increase in D-pyr (marker of bone resorption). Clinically, enzyme therapy was associated with a progressive reduction in BMD in these splenectomized patients, although there was an improvement in lumbar spine fat fraction, an indication of the burden of Gaucher cells in the bone marrow. ${ }^{42}$

In patients treated consecutively with enzyme replacement therapy and substrate reduction therapy, osteocalcin and alkaline phosphatase were within the normal range (lower part of reference ranges) at the time of the change from enzyme replacement therapy to start of reduction therapy, and decreased during follow up of 12-28 months (statistically significant for alkaline phosphatase). There was no statistically significant change in markers of bone resorption (CTX, TRAP5b). Imaging parameters of bone disease (MRI, DEXA) generally remained stable throughout follow up, although one patient experienced worsening bone parameters observed by MRI. This patient also experienced increasing bone pain and demonstrated a significant increase in TRAP5b. ${ }^{41}$

\section{The effect of bisphosphonates on bone biomarkers in Gaucher disease}

In untreated patients with severe skeletal involvement, pamidronate therapy (plus calcium) resulted in a decrease in osteocalcin, hydroxyproline, total D-pry, and urinary calcium after 3-5 months of therapy compared to baseline. Pamidronate treatment also resulted in increased BMD. ${ }^{43}$

The second study involved GD patients who had all received enzyme replacement therapy for at least 24 months (plus vitamin D and calcium). Biomarkers were within normal reference range at baseline even in patients with severe osteopenia $(\mathrm{T}<-2)$. One group of patients received bisphosphonate therapy (alendronate) for 24 months. There was a significant reduction in osteocalcin and BSAP for a period of 18 months after the initiation of bisphosphonates but markers of bone resorp- tion were inconsistent with reduced NTXs, but not D-pyr links. There was a statistically significant increase in BMD in the alendronate treated group $(\mathrm{P}=0.001){ }^{44}$

\section{Studies involving mixed populations of patients (treated and untreated/type 3 Gaucher disease)}

Three studies looked at different mixed groups of patients: i) patients who had and those who had not been treated with enzyme therapy (and for varying periods of time); ii) patients who had been treated with other medications such as bisphosphonates; ${ }^{21,45}$ iii) patients who were diagnosed with different GD types (types 1 and 3 ) and who had been treated with enzyme replacement therapy for varying periods ( 1 month to over 11 years). ${ }^{31}$

Fiore et al. ${ }^{45}$ examined bone biomarkers in 12 patients, 10 of whom had received enzyme treatment for 2-8 years. Markers of bone formation were within normal ranges while the markers of bone resorption, pyr and D-pyr, were significantly increased over control values, leading the authors to conclude that bone resorption was up-regulated in GD. ${ }^{45}$

Magal et $a l .{ }^{21}$ were the only authors to examine the role of the RANKL/OPG system in GDrelated bone disease. They found no difference in serum OPG between GD patients and controls, and did not identify any correlation between OPG and disease severity. The authors concluded that OPG-related osteoclast activity was probably not a major underlying mechanism for bone disease in their cohort. The patients $(n=173)$ were described as having non-severe involvement and were heterogeneous in terms of age, spleen status and treatment (untreated, enzyme-treated for variable periods, or treated with bisphosphonates). ${ }^{21}$

A third study, which mainly focused on examining the role of cytokines in bone remodeling, involved both pediatric and adult patients: 4 with type 1 and 4 with type 3 disease. All had received enzyme therapy for 1-141 months. Osteocalcin and BSAP were found to be within normal ranges in adults, while BSAP was marginally elevated and osteocalcin was below the normal reference range in children. ${ }^{31}$

\section{Discussion}

Studies involving bone markers in GD cover a wide range of different study designs: patient groups in terms of the severity of bone disease, demographics, treatment regimens, and bone biomarkers. Such diversity means it is difficult to make a direct comparison between one study and another. Several studies suggest that bone turnover biomarkers may be disturbed in GD. ${ }^{32-35}$ However, the available evidence suggests variability in whether, and which, biomarkers are elevated or decreased in both untreated GD and treated GD patients (Tables 2 and 3).32-35,38-41 No one bone biomarker appears to reflect the presence of bone disease consistently across multiple studies of GD patients or the response to enzyme replacement therapy. This, in part, may have been related to the heterogeneity of GD. Patients may have differed in the severity of their disease and also in their bone manifestations, both within and between studies. Three pathological presentations may be associated with GD and often all three are presented together: i) focal lytic and/or sclerotic lesions as a result of infarction, thrombosis and inflammation; ii) local disease such as remodeling deformities and cortical bone thinning; and iii) generalized osteopenia and osteoporosis. ${ }^{10}$ It is not known whether, and to what extent, different pathological processes in GD-related bone disease might influence bone biomarker profiles, or how recent bone events might have affected overall results. Recent bone crises, for example, may result in increased levels of bone markers since bone crisis is associated with enhanced bone resorption. ${ }^{35}$ Similarly, bone markers may increase by $20-60 \%$ for periods of up to six months following a fracture. ${ }^{17}$

Most of the bone markers studied in GD have been used to assess the response to treatment in other disease states, notably osteoporosis. In these other conditions, a decline in bone biomarkers within weeks of starting bisphosphonate therapy indicates that anti-resorptive therapy is having an effect, even in the absence of noticeable changes in BMD, which may take several years. ${ }^{46}$ Studies in GD patients that measure biomarker response after the initiation of bisphosphonate therapies showed a decrease in bone biomarkers. A reduction in osteocalcin and hydroxyproline, ${ }^{43}$ and osteocalcin, BSAP and NTX, ${ }^{44}$ together with statistically significant increases in BMD suggest that bone markers may be responsive to bisphosphonate intervention in GD, and that the use of biomarkers may be relevant to the study of bisphosphonate treatment response. The observation that biomarker levels changed significantly in response to bisphosphonates in these studies also underlines the possible confounding effects of attempting to compare biomarkers in bisphosphonate-treated GD patients with controls and correlating biomarkers with clinical parameters in populations that include both bisphosphonate and non-bisphosphonate-treated patients. ${ }^{21,35}$ For example, Magel et al. ${ }^{21}$ found no differences in OPG levels between GD1 
patients, many of whom were treated with bisphosphonates, and healthy controls. In our own studies of the OPG/RANKL system in 5 enzymetreated GD patients who had not been exposed to bisphophonates, we found a statistically significant decrease in $\mathrm{OPG}$, and a consequent statistically significant decrease in the RANKL/OPG ratio ( $\mathrm{P}=0.0002)$ compared with age and gender matched controls. No statistically significant differences in the levels of PICP, osteocalcin or TRAP5b were found (Giuffrida $e t$ al., 2010, data not cited).

This review does not clarify whether biomarkers could be applied reliably to the measurement of the bone response to enzyme replacement therapy or SRT. Markers of bone formation, osteocalcin and BSAP increased by $60 \%$ in the first 12 months of enzyme treatment in one study with no significant change in markers of bone resorption, suggesting a shift towards new bone formation. This was reflected in an increase in vertebral and femoral neck BMD over time. ${ }^{39}$ Increased BMD has been demonstrated in another long-term study of enzyme-treated GD patients (who also received interrupted vitamin D supplementation). But in this study, there was no correlation between biomarker levels and BMD. ${ }^{38}$ Another study examining biomarker levels in a group of GD patients receiving enzyme therapy, all of whom had undergone splenectomy, demonstrated statistically significant increases in osteocalcin and BSAP $(\mathrm{P}=0.002$ and 0.008 , respectively) with only a transient increase in D-pyr. However, BMD decreased in enzyme-treated patients despite an apparent shift towards enhanced bone formation, as demonstrated by bone biomarker levels. ${ }^{40}$ Splenectomy in GD patients is associated with exacerbated bone disease thought to be related to an increased burden of Gaucher cells in bone. ${ }^{8,10}$ However, baseline data in splenectomized patients did not indicate that BSAP, osteocalcin and D-pyr were outside the normal reference range. ${ }^{40}$ Several other studies also included splenectomized patients in their cohorts. ${ }^{21,31,34,35,38,39,41,45,46}$ including the study by Sims et al. ${ }^{39}$ ( 5 of 33 patients, $15 \%$ splenectomized). But no author reported a significant difference in biomarker levels in these patients. These data tend to suggest that bone markers may not reflect skeletal changes that might occur as a consequence of splenectomy.

Unlike bisphosphonate therapy, where treatment is targeted directly at reducing bone resorption by a process believed to be related to increased osteoclast apoptosis, ${ }^{47}$ any effects of enzyme therapy (and SRT) on bone biomarkers are likely to be indirect and may occur through different mechanisms. The limited evidence of changes in bone markers in GD appears to suggest that bone markers tend to decrease in response to bisphosphonate thera- py (consistent with the response in non-GD populations) but tend to increase in response to enzyme therapy (Table 3).

Enzyme replacement therapy aims to reduce the burden of glucocerebroside in macrophages and thereby reduce Gaucher cell infiltration into organs. ${ }^{48}$ Glucocerebroside laden macrophages in bone tissue could have an impact on bone turnover by displacing bone cells, causing vascular occlusion, increasing intra-osseous pressure, and/or by influencing osteoblast and osteoclast activity through inflammatory mechanisms. Several studies have reported disturbances in the levels of serum cytokines in GD, including IL-1, IL-6 and TNF, ${ }^{49-51}$ which may affect osteoclasts, their function, and bone turnover. Indeed, Yoshino et al. ${ }^{31}$ found correlations between IL-18 and BSAP and osteocalcin, and M-CSF and urinary NTX, although in a limited number of enzyme-treated type $1 \quad(n=4)$ and type $3 \mathrm{GD}$ patients $(\mathrm{n}=4)$. A reduction in glucocerebroside burden, therefore, might be expected to impact bone turnover, but the temporal relationship between initiation of therapy and changes in different bone turnover markers has not been studied in detail. The rate of glucocerebroside clearance is likely to depend on multiple factors, such as vascularization, degree of osteonecrosis, fibrosis, and sanctuary sites in the bone in individual patients. ${ }^{52}$

The difficulties of determining whether and how biomarkers may be affected in GD, and their response to therapy, are compounded by the knowledge that bone biomarkers fluctuate according to age, diet, exercise, hormonal status, stage of the menstrual cycle, season, the use some medications (e.g. anticonvulsants, estrogen-based therapies, corticosteroids, insulin, fluoride, vitamin D supplements, calcitonin), immune factors, and inflammatory status, ${ }^{25,45,53}$ and that different biomarkers show different degrees of spontaneous and intraindividual variability. ${ }^{46,54}$ The response of markers of bone formation and bone resorption may also vary; bone resorption markers are reported to respond faster (2-12 weeks) than markers of formation (3-6 months). ${ }^{17}$ Urinary biomarkers may also show diurnal variation; PYR and D-pyr are reported to show 2-fold differences over a period of $24 \mathrm{~h},{ }^{55}$ and biomarker measurements may show inter-laboratory variation, even when identical assays and methods are used. ${ }^{56}$ This makes it challenging to detect meaningful changes in bone marker levels. Despite this, statistically significant correlations between biomarker levels and several measures of clinical bone status have been demonstrated in GD in one study. ${ }^{35}$

One of the limitations of the studies reviewed here is that bone marker levels are often not the study end point, and data on bone biomarkers were of secondary interest. Also, these data were captured in patients who were clinically and demographically heterogeneous. There have been no detailed studies of changes in biomarker levels over time in untreated and treated GD and their relationship with bone manifestations, leaving clinicians with very little guidance on which biomarkers might be useful in clinical practice.

The ideal biomarker in GD should: i) accurately reflect the presence and activity of disease; ii) predict clinically meaningful outcomes; iii) change rapidly with the response to therapy; iv) be easily measured and stable in accessible clinical samples; v) be specific and sensitive; vi) be subject to little or no genetic variation; vii) be reproducible according to agreed standards of measurement; and viii) be inexpensive to measure. ${ }^{8}$ These criteria are just as applicable to biomarkers specific for bone. Evidence from currently available studies does not suggest that any one bone biomarker, or group of biomarkers, reliably reflects the presence and/or activity of disease or changes in a predictable manner to enzyme replacement therapy. Bone biomarkers may also be subject to considerable variation as a result of patient status (hormonal, medications, etc.) or as a result of inter-laboratory variability. Bone markers reflect activity in the whole skeleton and may not be specific or sensitive enough to detect subtle localized changes over a general background of activity. In addition, most studies tend to report biomarker levels as being indicative of either bone formation or bone resorption, although some markers may be released as a consequence of both processes. Osteocalcin, for example, is produced by mature osteoblasts in the bone matrix, but is also released from the bone matrix during resorption. . $^{17,25}$

There is no evidence as to whether bone markers might have any predictive value in terms of the progression of bone disease or the need for treatment. A greater understanding of the temporal relationship between bone biomarkers and clinical consequence is required.

The difficulties of measuring biomarkers are compounded in rare diseases, such as GD, where few patients are available for study and where bone disease may reflect multiple pathological mechanisms. Future advances in identifying meaningful biomarkers of bone disease are, therefore, likely to depend on large internationally coordinated studies. Such studies might be able to compare carefully controlled groups of patients in sufficient numbers for meaningful statistical analyses to be made. Key questions to address include the temporal relationship between fluctuations in bone biomarkers and clinical disease. Do biomarkers measured today reflect current bone abnormalities or do they reflect an ongoing pathological process, the consequences of which are not yet measurable by DEXA, MRI or other commonly used monitoring methods? 


\section{Conclusions}

Studies involving bone biomarkers in GD show variable results which do not currently support their routine use for the clinical assessment of bone status, as an indication for therapy initiation, or for monitoring the response to therapy. A greater understanding of bone markers and their relation to the bone manifestations of GD is required.

\section{References}

1. Beutler E, Grabowski GA. Gaucher disease. In: Scriver CR, Beaudet AL, Sly WS, Valle D. The metabolic and molecular basis of inherited diseases. New York: McGrawHill; 2001. pp. 3635-3668.

2. Weinreb N, Barranger J, Packman S, et al. Imiglucerase (Cerezyme) improves quality of life in patients with skeletal manifestations of Gaucher disease. Clin Genet 2007; 71:576-88.

3. Vom Dahl S, Poll L, Di Rocco M, et al. Evidence-based recommendations for monitoring bone disease and the response to enzyme replacement therapy in Gaucher patients. Curr Med Res Opin 2006;22:1045-64.

4. Mistry PK, Weinreb NJ, Kaplan P, et al. Osteopenia in Gaucher disease develops early in life: response to imiglucerase enzyme therapy in children, adolescents and adults. Blood Cells Mol Dis 2011;46:6672 .

5. Grabowski GA, Barton NW, Pastores G, et al. Enzyme therapy in type 1 Gaucher disease: comparative efficacy of mannoseterminated glucocerebrosidase from natural and recombinant sources. Ann Intern Med 1995;122:33-9.

6. Weinreb NJ, Barranger JA, Charrow J, et al. Guidance on the use of miglustat for treating patients with type 1 Gaucher disease. Am J Hematol 2005;80:223-9.

7. Elstein D, Cohn GM, Wang N, et al. Early achievement and maintenance of the therapeutic goals using velaglucerase alfa in type 1 Gaucher disease. Blood Cells Mol Dis 2011;46:119-23.

8. Cox TM, Aerts JM, Belmatoug N, et al. Management of non-neuronopathic Gaucher disease with special reference to pregnancy, splenectomy, bisphosphonate therapy, use of biomarkers and bone disease monitoring. $\mathrm{J}$ Inherit Metab Dis 2008;31:319-36.

9. DeMayo RF, Haims AH, McRae MC, et al. Correlation of MRI-based bone marrow burden score with genotype and spleen status in Gaucher's disease. AJR
Am J Roentgenol 2008;191:115-23.

10. Pastores GM, Weinreb NJ, Aerts H, et al. Therapeutic goals in the treatment of Gaucher disease. Semin Hematol 2004; 41Suppl5:4-14.

11. Wenstrup RJ, Kacena KA, Kaplan P, et al. Effect of enzyme replacement therapy with imiglucerase on BMD in type 1 Gaucher disease. J Bone Min Res 2007;22:119-26.

12. Deegan PB, Pavlova E, Tindall J, et al. Osseous manifestations of adult Gaucher disease in the era of enzyme replacement therapy. Medicine (Baltimore) 2011;90:5260.

13. Andersson H, Kaplan P, Kacena K, Yee J. Eight-year clinical outcomes of long-term enzyme replacement therapy for 884 children with Gaucher disease type 1 . Pediatrics 2008;122:1182-90.

14. Mistry PK, Deegan P, Vellodi A, et al. Timing of initiation of enzyme replacement therapy after diagnosis of type 1 Gaucher disease: effect on incidence of avascular necrosis. Br J Haematol 2009; 147:561-70.

15. Weinreb NJ, Aggio MC, Andersson HC, et al. International Collaborative Gaucher Group (ICGG). Gaucher disease type 1: revised recommendations on evaluations and monitoring for adult patients. Semin Hematol 2004,41Suppl5:15-22.

16. Campeau PM, Rafei M, Boivin MN, et al. Characterization of Gaucher disease bone marrow mesenchymal stromal cells reveals an altered inflammatory secretome. Blood 2009;114:3181-90.

17. Watts NB. Clinical utility of biochemical markers of bone remodeling. Clin Chem 1999;45:1359-68.

18. Sims NA, Walsh NC. Intercellular crosstalk among bone cells: new factors and pathways. Curr Osteoporos Rep 2012;2: 109-17.

19. Boyce BF, Xing L. Osteoclasts have multiple roles in bone in addition to bone resorption. Crit Rev Eukaryot Gene Expr 2009;19:171-80.

20. Kearns AE, Khosla S, Kostenuik PJ. Receptor activator of nuclear factor kappaB ligand and osteoprotegerin regulation of bone remodeling in health and disease. Endocr Rev 2008;29:155-92.

21. Magal I, Lebel E, Altarescu G, et al. Serum levels of osteoprotegerin and osteoprotegerin polymorphisms in Gaucher disease. Br J Haematol 2006;133:93-7.

22. Vega D, Maalouf NM, Sakhaee K. Clinical Review: the role of receptor activator of nuclear factor-kappaB (RANK)/RANK ligand/osteoprotegerin: clinical implications. J Clin Endocrinol Metab 2007;92:4514-21.

23. Hamdy NA. Targeting the RANK/RANKL/ OPG signaling pathway: a novel approach in the management of osteoporosis. Curr
Opin Investig Drugs 2007,8:299-303.

24. Scott LJ, Muir VJ. Denosumab: in the prevention of skeletal-related events in patients with bone metastases from solid tumors. Drugs 2011;28:1059-69.

25. Singer FR, Eyre DR. Using biochemical markers of bone turnover in clinical practice. Cleve Clin J Med 2008;75:739-50.

26. Delmas PD, Hardy P, Garnero P, Dain M. Monitoring individual response to hormone replacement therapy with bone markers. Bone 2000;26:553-60.

27. Peris P, Alvarez L, Vidal S, et al. Treatment with tiludronate has a similar effect to risedronate on Paget's disease activity assessed by bone markers and bone scintigraphy. Clin Exp Rheumatol 2007;25: 206-10.

28. Karsdal MA, Schett G, Emery P, et al. IL-6 receptor inhibition positively modulates bone balance in rheumatoid arthritis patients with an inadequate response to anti-tumor necrosis factor therapy: biochemical marker analysis of bonemetabolism in the tocilizumab RADIATE study (NCT00106522). Semin Arthritis Rheum 2012 Mar 5. [EPub ahead of print].

29. Boumans MJ, Thurlings RM, Yeo L, et al. Rituximab abrogates joint destruction in rheumatoid arthritis by inhibiting osteoclastogenesis. Ann Rheum Dis 2012;71:108-13.

30. Loftus LS, Edwards-Bennett S, Sokol GH. Systemic therapy for bone metastases. Cancer Control 2012;19:145-53.

31. Yoshino M, Watanabe Y, Tokunaga Y, et al. Roles of specific cytokines in bone remodeling and hematopoiesis in Gaucher disease. Pediatr Int 2007;49:959-65.

32. Stowens DW, Teitelbaum SL, Kahn AJ, Barranger JA. Skeletal complications of Gaucher disease. Medicine (Baltimore) 1985;64:310-22.

33. Drugan C, Jebeleanu G, Grigorescu-Sido P, et al. Biochemical markers of bone turnover as tools in the evaluation of skeletal involvement in patients with type 1 Gaucher disease. Blood Cells Mol Dis 2002;28:13-20.

34. Ciana G, Martini C, Leopaldi A, et al. Bone marker alterations in patients with type 1 Gaucher disease. Calcif Tissue Int 2003;72: 185-9.

35. van Dussen L, Lips P, Everts VE, et al. Markers of bone turnover in Gaucher disease: modeling the evolution of bone disease. J Clin Endocrinol Metab 2011;96: 2194-205.

36. Zimran A, Kay A, Gelbart T, et al. Gaucher disease. Clinical, laboratory, radiologic, and genetic features of 53 patients. Medicine (Baltimore) 1992;71:337-53.

37. Hermann G, Pastores GM, Abdelwahab IF, Lorberboym AM. Gaucher disease: assess- 
ment of skeletal involvement and therapeutic responses to enzyme replacement. Skeletal Radiol 1997;26:687-96.

38. Ciana G, Addobbati R, Tamaro G, et al. Gaucher disease and bone: laboratory and skeletal mineral density variations during a long period of enzyme replacement therapy. J Inherit Metab Dis 2005;28:723-32.

39. Sims KB, Pastores GM, Weinreb NJ, et al. Improvement of bone disease by imiglucerase (Cerezyme) therapy in patients with skeletal manifestations of type 1 Gaucher disease: results of a 48month longitudinal cohort study. Clin Genet 2008;73: 430-40.

40. Schiffmann R, Mankin H, Dambrosia JM, et al. Decreased bone density in splenectomized Gaucher patients receiving enzyme replacement therapy. Blood Cells Mol Dis 2002;28:288-96.

41. Mikosch P, Reed M, Baker R, et al. Changes of bone metabolism in seven patients with Gaucher disease treated consecutively with imiglucerase and miglustat. Calcif Tissue Int 2008;83:43-54.

42. Maas M, van Kuijk C, Stoker J, et al. Quantification of bone involvement in Gaucher disease: MR imaging bone marrow burden score as an alternative to Dixon quantitative chemical shift MR imaging-initial experience. Radiology 2003;229:554-61.

43. Ciana G, Cuttini M, Bembi B. Short-term effects of pamidronate in patients with Gaucher's disease and severe skeletal involvement. NEJM 1997;337-712

44. Wenstrup RJ, Bailey L, Grabowski GA, et al. Gaucher disease: alendronate disodium improves bone mineral density in adults receiving enzyme therapy. Blood 2004;104: 1253-7.

45. Fiore CE, Barone R, Pennisi P, et al. Bone ultrasonometry, bone density, and turnover markers in type 1 Gaucher disease. J Bone Miner Metab 2002;20:34-8.

46. Rosen HN, Moses AC, Garber J, et al. Utility of biochemical markers of bone turnover in the follow-up of patients treated with bisphosphonates. Calcif Tissue Int 1998;63:363-8.

47. Weinstein RS, Roberson PK, Manolagas SC. Giant osteoclast formation and longterm oral bisphosphonate therapy. N Engl J Med 2009;360:53-62.

48. Cox TM. Gaucher disease: clinical profile and therapeutic developments. Biologics 2010;4:299-313.

49. Allen MJ, Myer BJ, Khokher AM, et al. Proinflammatory cytokines and the pathogenesis of Gaucher's disease: increased release of interleukin- 6 and interleukin10. QJM 1997;90:19-25.

50. Lichtenstein M, Zimran A, Horowitz M. Cytokine mRNA in Gaucher disease. Blood Cells Mol Dis 1997;23:395-401.

51. Barak V, Acker M, Nisman B, et al. Cytokines in Gaucher's disease. Eur Cytokine Netw 1999;10:205-10.

52. de Fost M, van Noesel CJ, Aerts JM, et al. Persistent bone disease in adult type 1 Gaucher disease despite increasing doses of enzyme replacement therapy. Haematologica 2008;93:1119-20.

53. Redlich K, Smolen JS. Inflammatory bone loss: pathogenesis and therapeutic intervention. Nat Rev Drug Discov 2012;11: 234-50.

54. Panteghini M, Pagani F. Biological variation in bone-derived biochemical markers in serum. Scand J Clin Lab Invest 1995;55:609-16.

55. Schlemmer A, Hassager C, Jensen SB, Christiansen C. Marked diurnal variation in urinary excretion of pyridinium crosslinks in premenopausal women. J Clin Endocrinol Metab 1992;74:476-80.

56. Seibel MJ, Lang M, Geilenkeuser WJ. Interlaboratory variation of biochemical markers of bone turnover. Clin Chem 2001;47:1443-50. 